Research Article

\title{
Efficient Data Transmission Based on a Scalar Chaotic Drive-Response System
}

\author{
Ang Li and Cong Wang \\ School of Software Engineering, Beijing University of Posts and Telecommunications, Beijing 100876, China \\ Correspondence should be addressed to Cong Wang; wangc@bupt.edu.cn
}

Received 14 November 2016; Accepted 28 December 2016; Published 31 January 2017

Academic Editor: Liu Yuhong

Copyright ( 2017 Ang Li and Cong Wang. This is an open access article distributed under the Creative Commons Attribution License, which permits unrestricted use, distribution, and reproduction in any medium, provided the original work is properly cited.

Based on a scalar chaotic drive-response system, an efficient big data transmission scheme has been presented in this paper. In our method, the sender can modulate a great quantity of messages in the drive system using Walsh function, and the receiver can recover the original data using our proposed efficient reconstruction algorithm. To explore the feasibility and effectiveness, a series of simulations are performed and the results show that our proposed scheme outperforms some traditional approaches. This scheme has some potential applications in chaotic laser communication.

\section{Introduction}

Big data brings people much convenience as well as many problems. In the area of big data, data is the carrier of information, and the exchange of information cannot be separated from the transmission of data. Therefore, the problem of big data secure transmission becomes very serious and cannot be avoided $[1,2]$. In recent years, chaotic secure communication has been one of the research focuses in the field of communication [3,4]. Because of the remarkable contribution of Pecora and Carroll who addressed the synchronization of chaotic systems using a drive-response conception [5], the research on chaotic secure communication based on chaotic synchronization attracted wide attention and gradually infiltrated to many other subjects [6-11]. In fact, the dynamic behavior of chaotic system has some properties, such as initial sensitivity and unpredictability. These excellent properties have led to some applications of chaotic synchronization, such as chaos masking [12-14], chaos shift keying $[15,16]$, and chaotic modulation [17-20]. In recent years, a large number of improved chaotic communication models have emerged, such as the combination of chaos communication and multiplexing technology [21, 22], wireless chaotic communication [23], ultrawideband chaotic communication [24], chaotic laser communication [25, 26], and chaotic communication scheme based on wave recorder and time delay [27]. Recently, one significant topic of chaotic communication mainly focuses on the time series analysis [28-30]. But how many messages can be transmitted by one scalar chaotic signal? In our previous work [31], we have already achieved multiple information transmission only using one scalar chaotic time series; however, in that scheme, the original data is modulated into the system parameters directly which limits the maximum quantity of transmitted information data.

The contribution of this paper lies in the following aspects. First, a novel multiple time-delay chaotic communication scheme for big data transmission is designed based on Walsh function by which a huge amount of information can be modulated into a chaotic system. Specifically, the sender integrates multiple original information into single information by using Walsh function and then modulates such integrated information into the parameters of the drive system. Next, we design an adaptive parameter estimation scheme to recover the integrated information. That is to say, the receiver can use the inverse mapping of Walsh function to recover the original information. At last we investigate the maximum amount of information carried by a scalar chaotic drive-response system. Based on Shannon's channel capacity theorem, because of the channel bandwidth and noise, there 
exists a boundary of the maximum information in a real communication channel $[32,33]$. To explore the boundary of maximum transmittable information, we perform extensive simulations and find that our scheme is much more effective than the traditional technologies.

The remainder of this paper is structured in the following manner. We introduce the mathematical proof of the chaotic synchronization and the parameter adaptive estimation criterion in Section 2. Section 3 describes the design of chaotic communication scheme based on Walsh function and demonstrates the information recovery algorithm. In Section 4, the experimental results are showed to find out the maximum number of information carried by our scheme. Section 5 analyzes the application of our scheme. Finally, we draw our conclusions in Section 6.

Some symbols are used in this paper which are presented in Notations.

\section{The Adaptive Synchronization Scheme}

In this paper, we study the efficient data transmission using a scalar chaotic signal. For this purpose, we design a system model to carry as much information as possible. Based on the Mackey-Glass system [34], we consider a scalar time-delay chaotic system as follows:

$$
\dot{x}(t)=-\alpha x(t)+\frac{\beta x(t-\tau)}{1+x^{\gamma}(t-\tau)}+\sum_{i=1}^{m} a_{i} x\left(t-\tau_{i}\right),
$$

where $x(t)$ denotes the state variable of the system, $\alpha, \beta$, and $\gamma$ are constants, and $\tau, \tau_{i}$ are the time delays. $a_{1}, a_{2}, \ldots, a_{m}$ are system parameters which represent the original messages in this paper. Therefore, the bigger $m$ is, the more information the system can carry. In this model, we can adjust the amount of information carried by the system by changing the time delays $\tau, \tau_{i}$.

Based on the system in (1), a communication scheme is proposed. As the information is modulated in the system parameters, we make use of the parameter estimation method to get the recovered information. Based on synchronization principle, we design the following response system and the adaptive criterion:

$$
\begin{aligned}
\dot{y}(t)= & -\alpha y(t)+\frac{\beta x(t-\tau)}{1+x^{\gamma}(t-\tau)}+\sum_{i=1}^{m} \widehat{a}_{i} x\left(t-\tau_{i}\right) \\
& +u(t), \\
u(t)= & -(\eta+\alpha) e(t), \\
\dot{\hat{a}}_{i}= & -e(t) x\left(t-\tau_{i}\right),
\end{aligned}
$$

where $\hat{a}$ is the estimated parameter, $u(t)$ is the controller, and $\eta$ is a positive constant. $e(t)$ denotes the error term, which can be defined as $e(t)=y(t)-x(t)$. According to the drive system and the response system, the error system can be written as

$$
\dot{e}(t)=-\eta e(t)+\sum_{i=1}^{m}\left(\widehat{a}_{i}-a_{i}\right) x\left(t-\tau_{i}\right) .
$$

To verify that the estimated parameter $\widehat{a}_{i}$ converges to the original system's parameters, we present the proof as follows.

The Lyapunov function $V(t)$ is constructed as

$$
V(t)=\frac{1}{2} e^{2}(t)+\frac{1}{2} \sum_{i=1}^{m}\left(\widehat{a}_{i}-a_{i}\right)^{2} .
$$

The time derivative of $V(t)$ along the trajectories of (4) is described as follows:

$$
\begin{aligned}
\dot{V}(t)= & e(t) \dot{e}(t)+\sum_{i=1}^{m}\left(\widehat{a}_{i}-a_{i}\right)\left(\dot{\hat{a}}_{i}-\dot{a}_{i}\right) \\
= & -\eta e^{2}(t)+e(t) \sum_{i=1}^{m}\left(\widehat{a}_{i}-a_{i}\right) x\left(t-\tau_{i}\right) \\
& +\sum_{i=1}^{m}\left(\widehat{a}_{i}-a_{i}\right) \dot{\hat{a}}_{i} \\
= & -\eta e^{2}(t)+e(t) \sum_{i=1}^{m}\left(\widehat{a}_{i}-a_{i}\right) x\left(t-\tau_{i}\right) \\
& +\sum_{i=1}^{m}\left(\widehat{a}_{i}-a_{i}\right)\left(-e x\left(t-\tau_{i}\right)\right)=-\eta e^{2}(t) \leq 0 .
\end{aligned}
$$

Obviously, $\dot{V}=0$ if and only if $e=0$. From Barbalat's lemma, we can easily get $e \rightarrow 0$ and $\left(\dot{\hat{a}}_{i}-\dot{a}_{i}\right) \rightarrow 0$ as $t \rightarrow$ $\infty$. Thus, we can acquire the largest invariant set $M$ which is defined as $M=\left\{e \in R^{n},\left(\widehat{a}_{i}-a\right) \in R^{m} \mid e=0,-e+\sum_{i=1}^{m}\left(\widehat{a}_{i}-\right.\right.$ $\left.\left.a_{i}\right) x\left(t-\tau_{i}\right)=0\right\}$. In this case, the following equation can be satisfied:

$$
\sum_{i=1}^{m}\left(\widehat{a}_{i}-a_{i}\right) x\left(t-\tau_{i}\right)=0
$$

Let $D(x)=\left\{x\left(t-\tau_{1}\right), x\left(t-\tau_{2}\right), \ldots, x\left(t-\tau_{m}\right)\right\}, \widehat{A}=$ $\left(\widehat{a}_{1}, \widehat{a}_{2}, \ldots, \widehat{a}_{m}\right)^{T}$, and $A=\left(a_{1}, a_{2}, \ldots, a_{m}\right)$. Then, (6) can be written as follows:

$$
D(x)(\widehat{A}-A)=0 .
$$

Then both sides of (7) are multiplied by $D(x)^{T}$ and integrated for any period of time $\sigma$, and we get the following:

$$
\int_{s}^{s+\sigma} D(x)^{T} D(x)(\widehat{A}-A) d t=0 .
$$

Let $G=\int_{s}^{s+\sigma} D^{T}(x(t)) D(x(t)) d t$. $G$ is called the Gram matrix of $D(x)$. Then we get $G(\widehat{A}-A)=0$. If $G$ has full rank, (8) has a unique zero solution $[35,36]$. That is to say; $\widehat{A}-A=0$, that is, $\widehat{a}_{i}=a_{i}$. The proof of the synchronization and estimation criterion for the chaotic system is completed.

\section{The Walsh-Based Transmission Scheme}

In this section, we design a transmission scheme based on Walsh function which can further increase the maximum 


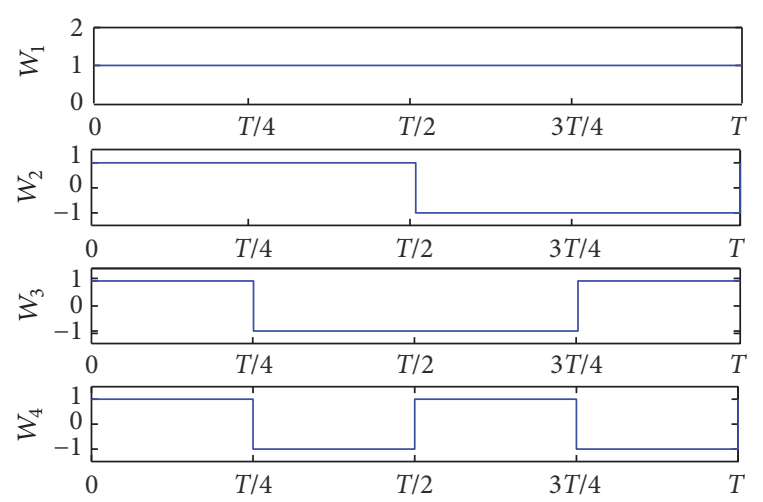

Figure 1: The 4-order Walsh Function.

quantity of transmitted information. The Walsh function is a kind of nonsinusoidal orthogonal complete function set [37]. A 4-order Walsh function is depicted in Figure 1.

It is easy to find that the elements of Walsh function set fully satisfy the orthogonality with each other. Note that as the number of available sequences is very large, it satisfies the demand of multiple information transmission.

Based on the properties of Walsh function, we consider a system based on the Mackey-Glass system; the drive system (1) can be redesigned as follows:

$$
\dot{x}(t)=f(x(t))+\sum_{i=1}^{m} \sum_{j=1}^{k}\left(a_{i j} W_{j}(t)\right) x\left(t-\tau_{i}\right),
$$

where $f(x(t))=-\alpha x(t)+\beta x(t-\tau) /\left(1+x^{\gamma}(t-\tau)\right), a_{i j}$ is the transmitted original message, and $W_{j}(t)$ is the $j$ th Walsh function among $k$-orders Walsh function. In this way, there are $k$ original messages in each system parameter. Therefore, the number of message increases from $m$ to $m \times k$.

We introduce the following formula to measure the total number of messages carried by this scheme:

$$
\mathrm{Q}=\frac{H}{l_{b}}(m \times k),
$$

where $Q$ denotes the quantity of total information (bits) carried by the system, $H$ is the effective length of the carrier, $l_{b}$ represents the length of one bit of information, and $m$ and $k$ are the number of the system parameters and the orders of Walsh function, respectively.

The corresponding response system and the adaptive criterion can be designed as follows:

$$
\begin{aligned}
\dot{y}(t) & =f(y(t))+\sum_{i=1}^{m} \widehat{b}_{i} x\left(t-\tau_{i}\right)+u(t), \\
u(t) & =-(\eta+\alpha) e(t), \\
\dot{\vec{b}}_{i} & =-e(t) x\left(t-\tau_{i}\right),
\end{aligned}
$$

where $f(y(t))=-\alpha y(t)+\beta x(t-\tau) /\left(1+x^{\gamma}(t-\tau)\right)$ and $\widehat{b}_{i}$ is the estimated information of the system parameters. As we already proved the synchronization of the system, similarly, the system presented in (9) can also be synchronized by following the same procedure.

Theoretically, the estimated parameters converge to the true value when $t \rightarrow \infty$. However, in practical scenarios, it requires a very short time. More precisely, the estimated parameters take a transient time to approach the true values and after that they remain unchanged. Thus, if we set up a sampling point at each unchanged period and then design a threshold mechanism to distinguish the estimated parameters, we get the estimated system parameters precisely. Based on (11), as $a_{i j}$ is binary, thus $a_{i j} W_{j}(t)$ must be integral; the threshold mechanism can be designed as follows:

$$
\begin{aligned}
& \text { output }=n, \quad \text { if } \frac{2 n-1}{2} \leq\left.\widehat{b}_{i}\right|_{t_{j}}<\frac{2 n+1}{2} ; \\
& \text { output }=0, \quad \text { if }-0.5 \leq\left.\widehat{b}_{i}\right|_{t_{j}}<0.5 ; \\
& \text { output }=-n, \quad \text { if } \frac{-2 n-1}{2} \leq\left.\widehat{b}_{i}\right|_{t_{j}}<\frac{-2 n+1}{2},
\end{aligned}
$$

where $n=1,2,3, \ldots, k, t_{j}=j l_{w}$ is the sample time and $l_{w}=l_{b} / k$ denotes the length of $k$-orders Walsh function's symbol. Until the convergent time remains short enough for the threshold mechanism, we get $\widehat{b}_{i}=\sum_{j=1}^{k} a_{i j} W_{j}(t)$.

Next, we present the recovering algorithm of the Walsh function to recover the original information. We multiply $\widehat{b}_{i}$ by the corresponding Walsh function then integrate them for each period $T$ and thereby the original message is recovered. For example, if the information to be recovered is $a_{p q}(1 \leq$ $p \leq m, 1 \leq q \leq k)$, then the estimated information is $\hat{b}_{p}$. As we proved before, $\widehat{b}_{p}=\sum_{j=1}^{k} a_{p q} W_{j}(t)$. The process of calculation is presented as follows:

$$
\begin{gathered}
\int_{(\theta-1) T}^{\theta T} \widehat{b}_{p} W_{q}(t) d t=\int_{(\theta-1) T}^{\theta T}\left[\sum_{j=1}^{k} a_{p q} W_{j}(t)\right] W_{q}(t) d t \\
=a_{p q} \int_{(\theta-1) T}^{\theta T} W_{q}(t)^{2} d t \\
+a_{p q} \sum_{j=1, j \neq q}^{k} \int_{(\theta-1) T}^{\theta T} W_{j}(t) W_{q}(t) d t=a_{p q}, \\
(\theta=0,1,2,3, \ldots) .
\end{gathered}
$$

Remark 1. Step 2 and step 3 of (13) are using the property of Walsh function that

$$
\int_{0}^{T} W_{i}(t) W_{j}(t) d t=\left\{\begin{array}{ll}
0, & i \neq j, \\
1, & i=j,
\end{array} \quad(i, j \in k) .\right.
$$

As a result $\int_{(\theta-1) T}^{\theta T} W_{q}(t)^{2}=1$ and $\int_{(\theta-1) T}^{\theta T} W_{j}(t) W_{q}(t)=0$.

Thus, a chaotic communication model that combines the Walsh function and the adaptive parameter identification technique is finally obtained. Thus far, the Walsh-based transmission scheme has been established. The main process is presented in Figure 2. 


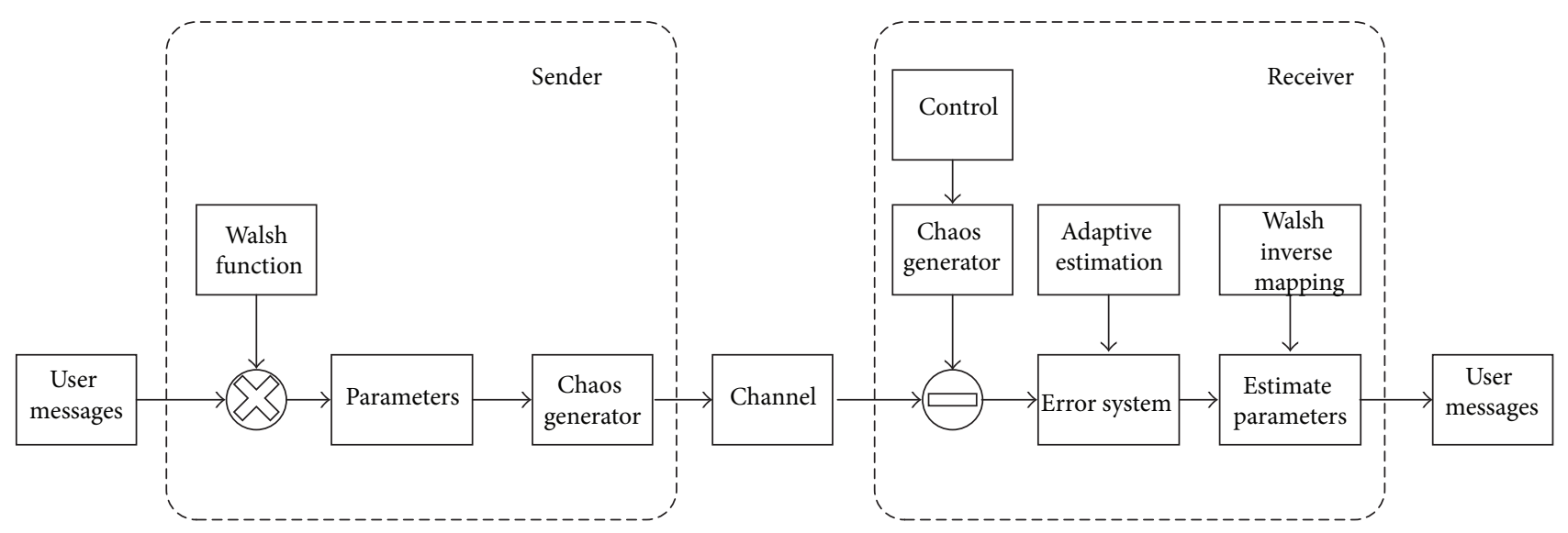

FIgURE 2: The flowchart of a procedure of communication.

Remark 2. We present some comparisons between different communication schemes on the total amount of messages. First, in our scheme, plenty of messages can be made into one mixed message, furthermore, many such mixed messages be can modulated into a multiple time-delay system; thus in our scheme the quantity of messages carried by the system is very huge $(m \times k=960)$. In the chaos masking scheme, only one carrier of message is carried by the chaotic system, that is, $m=1$. In the chaotic modulation scheme, the value of $m$ depends on the system's dimensions as the messages are modulated into the system; thus the chaotic system will be very complex. In the chaotic shift keying scheme, $m$ equals the number of the system parameters which steal less than ours. Compared with these communication schemes, our scheme strongly increases the total amount of messages carried by the chaotic system. In addition, our scheme uses a scalar chaotic signal which makes it easier to produce and transmit.

\section{Experiment and Simulation}

In this section, we will explore the maximum quantity of transmitted information by our scheme. At first, we consider a system based on the Mackey-Glass model as presented below:

$$
\begin{aligned}
& \dot{x}=f(x(t))+\sum_{i=1}^{m} \sum_{j=1}^{k}\left(a_{i j} W_{j}(t)\right) x\left(t-\tau_{i}\right), \\
& \dot{y}=f(y(t))+\sum_{i=1}^{m} \widehat{b}_{i} x\left(t-\tau_{i}\right),
\end{aligned}
$$

where $f(x(t))=-1100 x(t)+50000 x(t-\tau) /\left(1+x^{20}(t-\tau)\right)$, $f(y(t))=-1100 y(t)+50000 x(t-\tau) /\left(1+x^{20}(t-\tau)\right)$, and $\alpha=-1100, \beta=50000, \gamma=20, \tau=0.5, \tau_{i}=1+0.1 i . a_{i j}$ is the original information represented as random binary sequence with arbitrary length. In the simulation, we set up the relative tolerance to $1 \times e^{-4}$.

Remark 3. To ensure the chaotic property of the system, we attempt to adjust the values of $\alpha, \beta$, and $\gamma$ appropriately. We have set different values of $\alpha, \beta$, and $\gamma$ to start simulation, and at last we find the system has an excellent chaotic property when $\alpha=-1100, \beta=50000, \gamma=20$, and $\tau=0.5$.

4.1. Simulation with Different $m$. As the quantity of the transmitted information is determined by $m \times k$, we first choose $k=32$; that is, we use the 32-order Walsh function. Subsequently, we increase $m$ as required. For $m=20$, the corresponding results are shown in Figures 3(a)-3(f). Figure 3(a) displays the information combined by Walsh function. It forms an integral wave. The length of each bit is set to 0.2 ; that is, $l_{w}=0.2$. For making the original binary information to satisfy the orthogonal relation, the bit width of the original information is set to 6.4 ; that is, $l_{b}=6.4$. Since there is a block time $t_{\mathrm{bl}}=20$ for the running system from the initial state to the stable state, we cannot recover the information until $t \geq 20$; the effective length of the scalar series is taken as $H=180$. As mentioned before, the number of the system parameters is selected as $m=20$ and the order of Walsh function as $k=32$; thus, based on (10), the quantity of information loaded in the system is $Q=18000$.

Figure 3(b) shows that a scalar chaotic signal $x(t)$ is sent by the sender. Based on chaotic synchronization, we get the error signal as depicted in Figure 3(c). We observe that the synchronization error will converge to 0 for each sampling time $\left(t_{j}=0.2\right)$ from the details of $e(t)$. Hence, the estimated values converge to the value integrated by Walsh function in each sampling time as presented in Figure 3(d). We set up a sampling point at $t=0.2 j$. In this way, we can accurately estimate the accurate Walsh integrated information. After that, based on (13), we let the estimated value $\widehat{b}_{i}$ be multiplied with the corresponding Walsh function and then integrate them in one period of Walsh function. If the obtained original binary information is 1 , the result of the integral will be positive; otherwise, the result of integral will remain unchanged. Thus, we get a ladder-like waveform as presented in Figure 3(e). From that ladder-like waveform, we can recover the original binary information by using the 


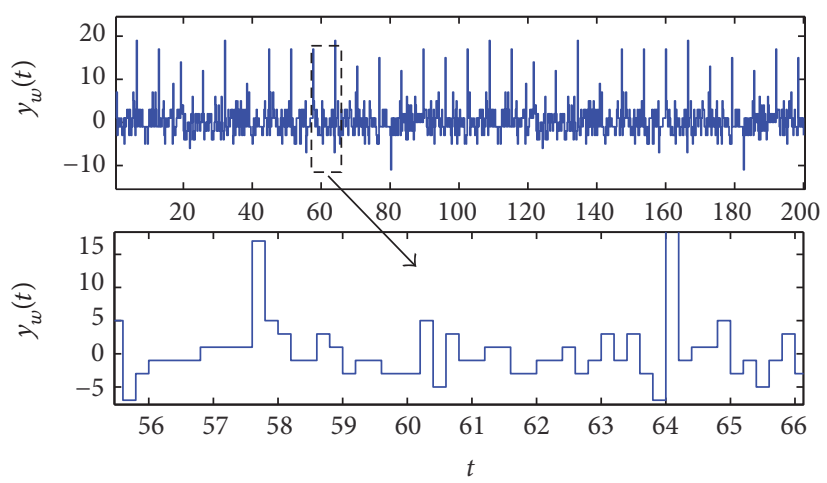

(a) The Walsh integrated message. $y_{w}(t)=\sum_{j=1}^{k} a_{i j} W_{j}(t)$

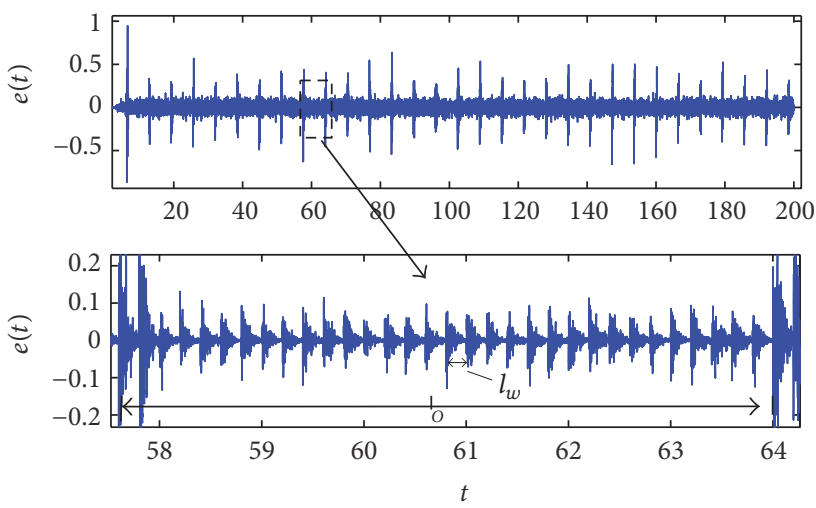

(c) The system error $e(t)$ when $m=20$ and $k=32$

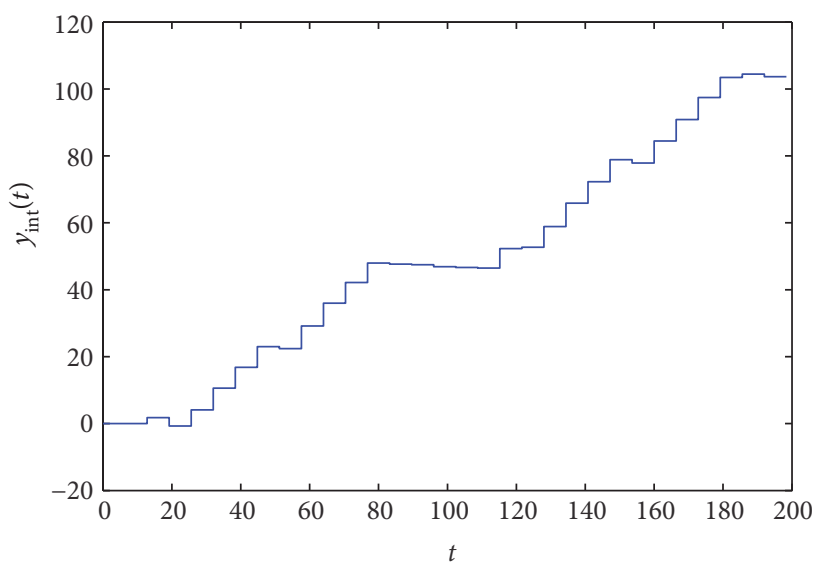

(e) The ladder-like trance of integral. $y_{\text {int }}(t)=\int_{(\theta-1) T}^{\theta T} \widehat{b}_{i} W_{j}(t) d t$. Each rising edge denotes the " 1 " and others denote the " 0 "

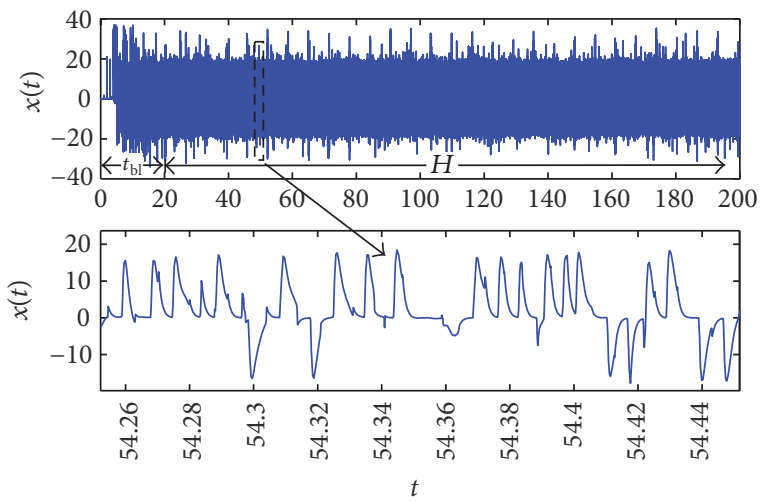

(b) The transmitted signal $x(t)$
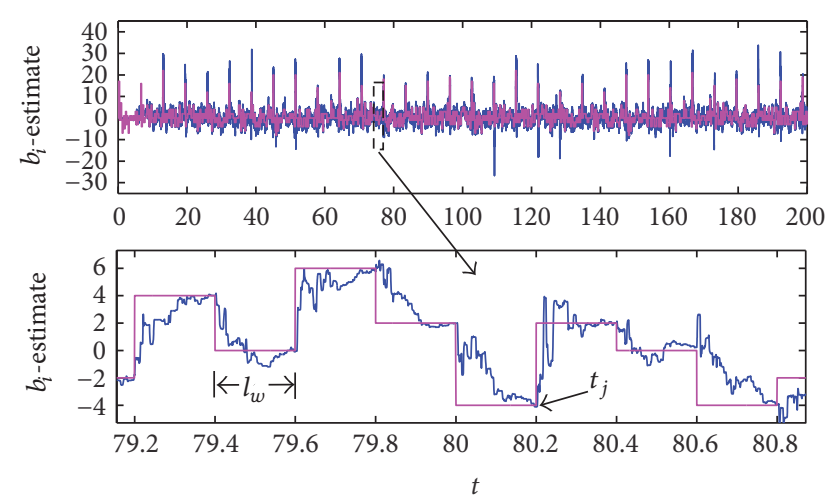

(d) The stochastic comparison of estimated value and the accurate value of the 20 Walsh integrated messages
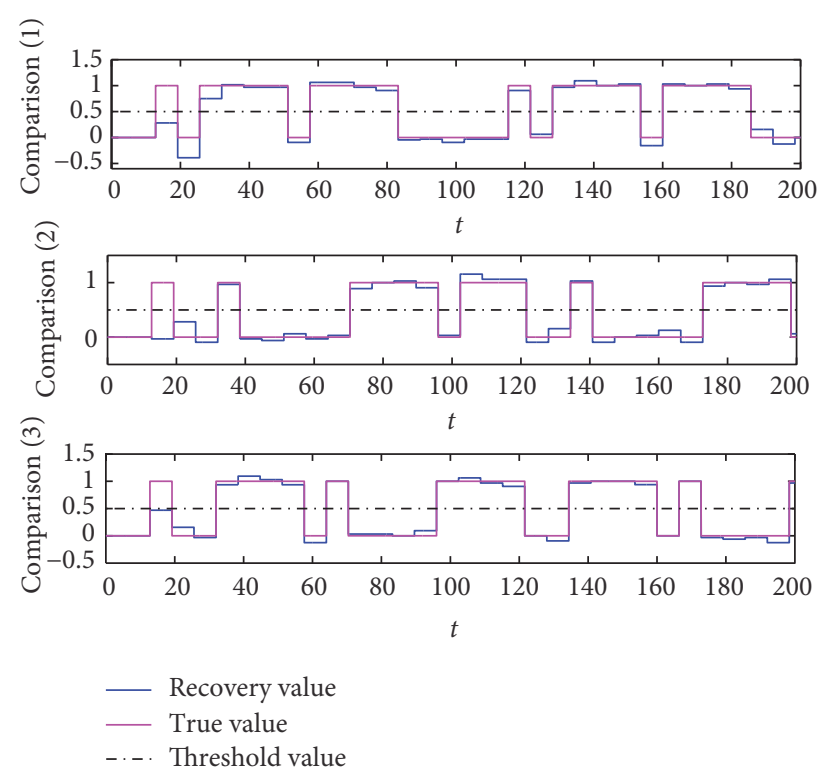

(f) The stochastic three comparisons of recovered value and original value

FIgURE 3: The simulation results when $k=32$ and $m=20$.

method that each rising edge equals " 1 " and others equal " 0 ." The comparisons of the recovered value and the original value are shown in Figure 3(f). We set a threshold $a_{\mathrm{th}}\left(a_{\mathrm{th}}=\right.$ 0.5 ); we can easily distinguish 0 and 1 . Thus, the transmitted information is precisely restored.
In the next step, we raise the value of $m$ to 30 . The results are depicted in Figures 4(a)-4(c). The error signal in Figure 4(a) is compared with Figure 3(c). It is obvious that the rate of convergence when $m=30$ is slower than that of when $m=20$. Thus, it points out to some minor 


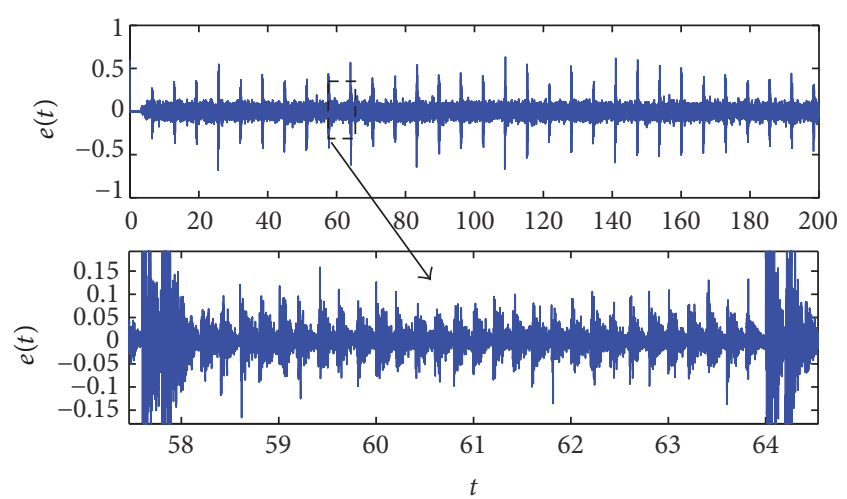

(a) The system error $e(t)$ when $m=30$ and $k=32$

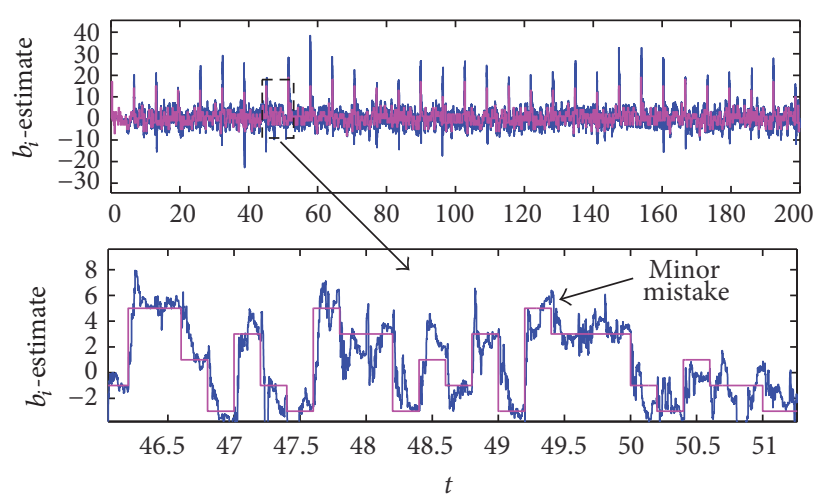

(b) The stochastic comparison of estimated value and the accurate value of the 30 Walsh integrated messages

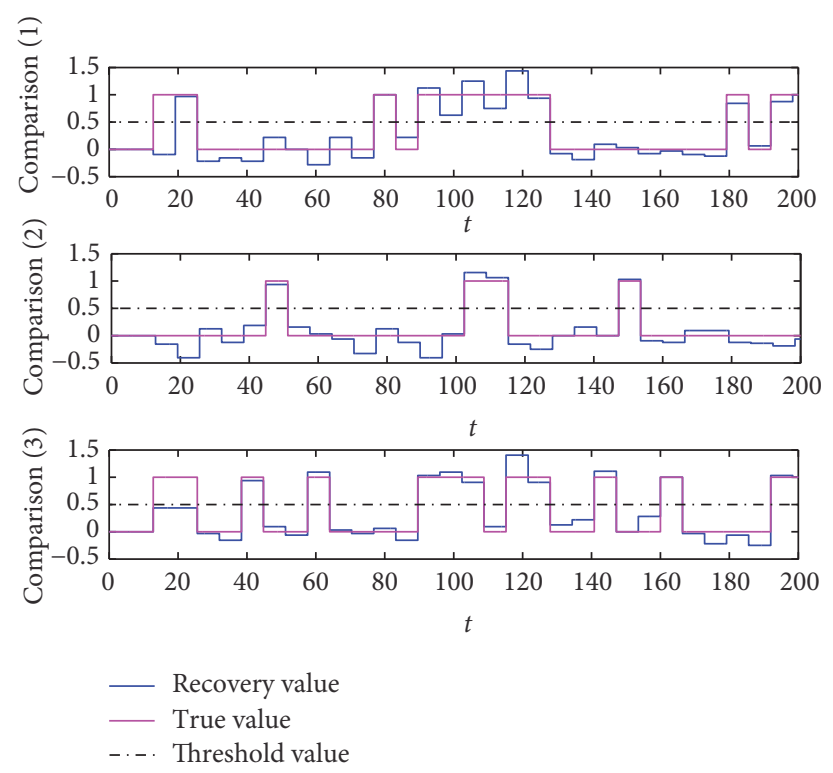

(c) The stochastic three comparisons of recovered value and original value

FIGURE 4: The simulation results of $k=32$ and $m=30$.

mistake in Figure 4(b). This minor mistake lies within the permitted sphere of estimation when $m=30$, so we can still recover the original information accurately. While compared with Figure 3(f), we find that the recovered information is far away from original value even almost beyond the threshold as presented in Figure 4(c). On the other hand, the recovered information lies near to the original value when $m=20$. With the increment of $m$, more and more errors appear in $\widehat{b}_{i}$ which becomes the hurdle to recover the original information. Under the premise of the accuracy, as a result, the experimental maximum of $m$ is 30. Thus, based on (10), the maximum quantity of information carried by the system is $Q=27000$. This quantity of information is much larger than that of traditional chaotic communication schemes.

4.2. Simulation with Different $k$. Next, we change the order of the Walsh function while fixing the width of original information to $l_{b}=6.4$ which is the same as $k=32$.
TABLE 1: The total information for different $k$.

\begin{tabular}{lcccc}
\hline$k$ & 8 & 16 & 32 & 64 \\
\hline$l_{w}$ & 0.8 & 0.4 & 0.2 & 0.1 \\
$l_{b}$ & 6.4 & 6.4 & 6.4 & 6.4 \\
$m_{\max }$ & 115 & 58 & 30 & 14 \\
$H$ & 180 & 180 & 180 & 180 \\
$Q$ (bit) & 25875 & 26100 & 27000 & 25200 \\
\hline
\end{tabular}

Under the premise that the system can accurately recover the original information, we let $k=8,16,64$ and simulate the experiments for each case separately. The results are presented in Table 1.

We expect the system can carry information as much as possible, but we observe from Notations that $m$ decreased as $k$ increases. Thus, we cannot increase $m$ and $k$ at the same time. Meanwhile, the total information presents a small uptrend when $l_{w} \geq 0.2$ and then goes down. Thus we get the 


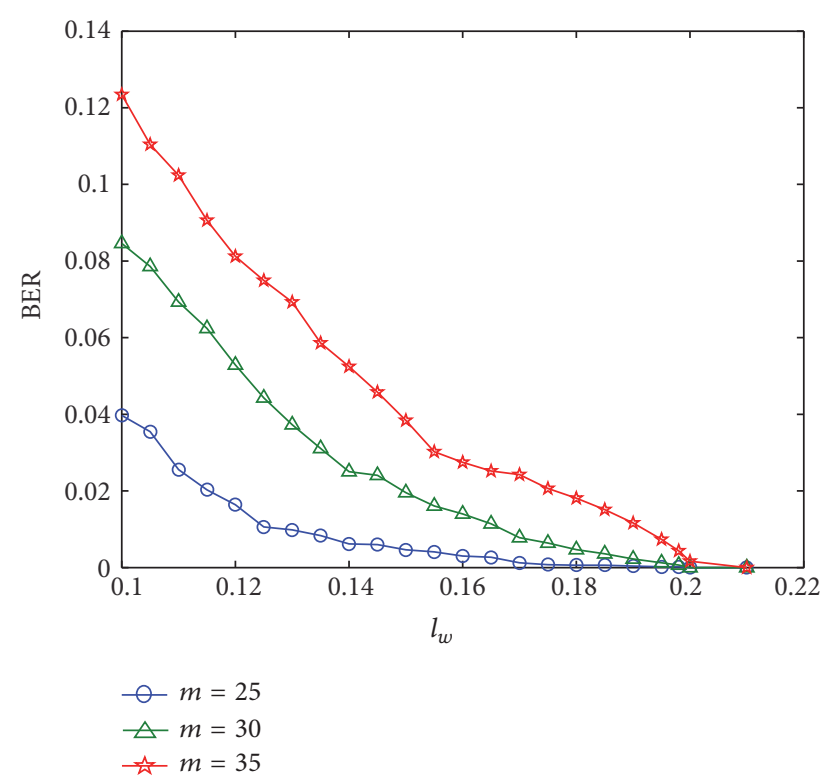

FIGURE 5: The bit error rate (BER) in different $l_{w}$ and $m$.

maximum information when $k=32$, and the total number of information is 27000 . That is the reason to set $k=32$ for the simulation at the beginning of this section.

Remark 4. Why choose $l_{w}=0.2$ ? To explain this question, we perform a series of simulations with different $l_{w}$ under 32order Walsh function. The result is depicted in Figure 5. We observe from here that the BER decreases with the increment of $l_{w}$. Thus, the smaller $l_{w}$ is, the more information the system could carry. We expect $l_{w}$ to be as small as possible, but it should be long enough so that the estimated value can converge to the true value. Thus, under the condition of non$\mathrm{BER}$, the minimum of $l_{w}$ is set to 0.2 .

4.3. Simulation with Gaussian White Noise. Next, the effect of noise is under consideration in our system. We add an Gaussian white noise in the drive system which can be written as follows:

$$
\dot{x}(t)=f(x(t))+\sum_{i=1}^{m} \sum_{j=1}^{k}\left(a_{i j} W_{j}(t)\right) x\left(t-\tau_{i}\right)+G(t),
$$

where $G(t)$ denotes Gaussian white noise with its expectation and variance set to $(0,25)$. The result is shown in Figure 6. Despite such noise, the simulation still recover the original information. That is to say, our system has a good ability to resist system noise. As the variance of the Gaussian white noise increases, the recovery accuracy tends to decrease. In the case when the variance exceeds 29 , the nonerror recovery cannot be achieved.

\section{Application Analysis}

In the following section, considering the Shannon-Hartley theorem $[32,33]$, we analyze the relationship between the signal transmission rate and the signal power in the real channel. First, we present the formula of calculating the average power of signal $S$ as follows:

$$
S=\lim _{T \rightarrow \infty} \frac{1}{T} \int_{-\infty}^{\infty} x(t)^{2} d t
$$

By using the formula, we can calculate the average power of $x(t)$, when $k=32$, and $m$ is set to $m=1,10,20,30$. Then we let $\delta=m * k$; the relation after calculations is presented as follows: $S_{\delta=32}<S_{\delta=320}<S_{\delta=640}<S_{\delta=960}$. The Shannon-Hartley theorem describes the relationship between the upper bound for the rate of transmission of information in a real channel and the channel signal-to-noise ratio and bandwidth; thus, it indicates that different bandwidths of modern wireless systems cause different maximum throughput of single carrier. The formula to characterize the theorem is presented as follows:

$$
C=B \log \left(1+\frac{S}{N_{0} B}\right)
$$

where $C$ denotes the information transmission rate, $B$ is the bandwidth of the channel, and $N_{0}$ is the noise power. Given $B$ and $N_{0}$, the rate of transmission increases with the growth of the average power of signal; that is, $C_{\delta=32}<$ $C_{\delta=320}<C_{\delta=640}<C_{\delta=960}$. In the era of big data, chaotic laser communication has great potential for mass quantity data transmission. Based on the aforementioned analysis, we conclude that if our proposed model is applied to the real chaotic laser communication, as the number of transmission information in our scheme is much larger than the traditional chaotic technology under the same setup, the efficiency of chaotic laser communication can be improved. In recent years, the long-haul and low-cost chaotic optical secure communications with $1.25 \mathrm{Gbits} / \mathrm{s}$ message and $2.5 \mathrm{Gbits} / \mathrm{s}$-message are experimentally realized using discrete optical components. The transmission distance reaches $143 \mathrm{~km}$ and $25 \mathrm{~km}$ [38], which is based on chaotic masking. Since the transmission rate $C_{\delta=32}<C_{\delta=960}$ under the same position, if our technology is applied in the above real system, the overall rate can be further increased to some extent; we will discuss the related issues in future research.

\section{Conclusion}

In summary, for the purpose of big data transmission, an efficient chaotic communication scheme based on Walsh function is designed. Experimental simulations are performed to explore the maximum value of information carried by one-dimensional scalar chaotic signal and illustrate the feasibility of this scheme. Finally, the application is discussed and will be further studied in our future works. 


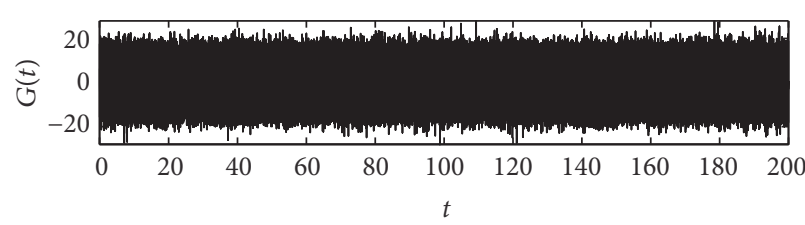

(a)

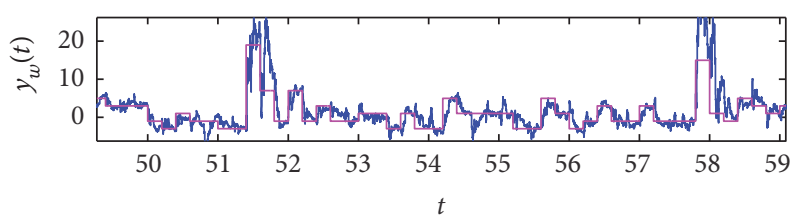

(c)

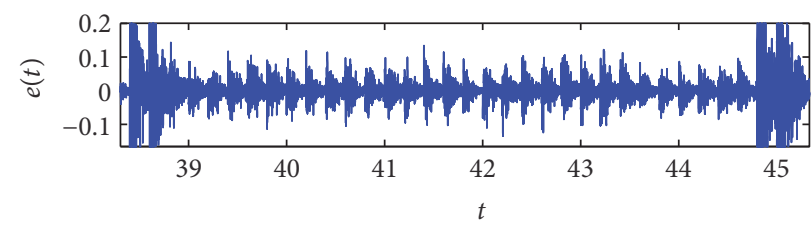

(b)

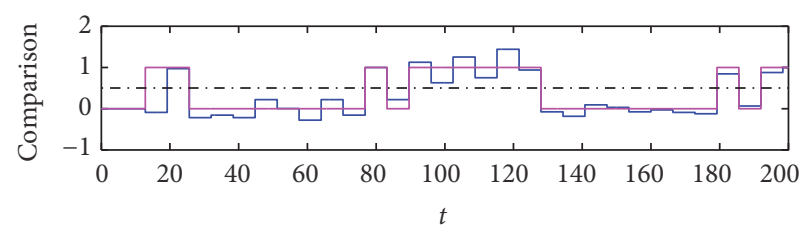

(d)

Figure 6: The simulation results of simulation with Gaussian white noise. (a) The noise $G(t)$ with its expectation and variance are set to $(0,25)$. (b) The system error $e(t)$ with noise when $m=30$ and $k=32$. (c) The comparison of estimated value and the accurate value of the 30 Walsh integrated messages with noise. (d) The comparisons of recovered value and original value in the 960 messages with noise.

\section{Notations}

$x(t): \quad$ The state variable of the drive system

$y(t): \quad$ The state variable of the response system

$e(t): \quad$ The state variable of the response system

$\tau, \tau_{i}: \quad$ The time delays

$a_{i}: \quad$ System parameters

$\widehat{a}_{i}: \quad$ The estimated value of $a_{i}$

$m$ : The number of system parameters

$\alpha, \beta, \gamma, \eta$ : Constants

$k$ : $\quad$ The order of Walsh function

$W_{j}(t): \quad$ The $j$ th Walsh sequence of $k$-orders Walsh function

$\widehat{b}_{i}: \quad$ The estimated value of $\sum_{j=1}^{k} a_{i j} W_{j}(t)$

$l_{b}$ : The length of one bit of information

$l_{w}$ : The length of each $k$-orders Walsh function's code element $\left(l_{w}=l_{b} / k\right)$

$t_{j}: \quad$ The sample time $\left(t_{j}=j l_{w}\right)$

$H$ : The effective length of the carrier

Q: $\quad$ The quantity of total information (bits) carried by the system.

\section{Competing Interests}

The authors declare that they have no competing interests.

\section{Acknowledgments}

The work is supported by the National Natural Science Foundation of China (Grant nos. 61472045 and 61573067), the National Key Research and Development Program (Grant no. 2016YFB0800602), the Beijing City Board of Education Science and Technology Key Project (Grant no. KZ201510015015), and the Beijing City Board of Education Science and Technology Project (Grant no. KM201510015009).

\section{References}

[1] J. Chen, Q. Liang, B. Zhang et al., "A new secure transmission for big data based on nested sampling and coprime sampling," in The Proceedings of the Second International Conference on Communications, Signal Processing, and Systems, pp. 733-741, Springer, 2014.

[2] J. Manyika, M. Chui, B. Brown et al., "Big data: the next frontier for innovation, comptetition, and productivity," Analytics, 2011.

[3] G. Kaddoum, E. Soujeri, and Y. Nijsure, "Design of a short reference noncoherent chaos-based communication systems," IEEE Transactions on Communications, vol. 64, no. 2, pp. 680689, 2016.

[4] M. F. Hassan, "Synchronization of uncertain constrained hyperchaotic systems and chaos-based secure communications via a novel decomposed nonlinear stochastic estimator," Nonlinear Dynamics, vol. 83, no. 4, pp. 2183-2211, 2016.

[5] L. M. Pecora and T. L. Carroll, "Synchronization in chaotic systems," Physical Review Letters, vol. 64, no. 8, pp. 821-824, 1990.

[6] T. Heil, I. Fischer, W. Elsässer, J. Mulet, and C. R. Mirasso, "Chaos synchronization and spontaneous symmetry-breaking in symmetrically delay-coupled semiconductor lasers," Physical Review Letters, vol. 86, no. 5, pp. 795-798, 2001.

[7] S. Hayes, C. Grebogi, and E. Ott, "Communicating with chaos," Physical Review Letters, vol. 70, no. 20, pp. 3031-3034, 1993.

[8] Y.-N. Li, L. Chen, Z.-S. Cai, and X.-Z. Zhao, "Study on chaos synchronization in the Belousov-Zhabotinsky chemical system," Chaos, Solitons \& Fractals, vol. 17, no. 4, pp. 699-707, 2003.

[9] C. Zhou and J. Kurths, "Dynamical weights and enhanced synchronization in adaptive complex networks," Physical Review Letters, vol. 96, no. 16, Article ID 164102, 2006.

[10] L. Li, H. Peng, X. Wang, and Y. Yang, "Comment on two papers of chaotic synchronization," Physics Letters A, vol. 333, no. 3-4, pp. 269-270, 2004.

[11] N. J. Corron and J. N. Blakely, "Chaos in optimal communication waveforms," Proceedings of the Royal Society A: Mathematical, Physical and Engineering Sciences, vol. 471, no. 2180, pp. 134-139, 2015.

[12] J. Gleick and R. C. Hilborn, "Making a new science," Physics Today, vol. 41, no. 11, p. 79, 1987.

[13] G. Álvarez, F. Montoya, M. Romera, and G. Pastor, "Breaking two secure communication systems based on chaotic masking," IEEE Transactions on Circuits and Systems II: Express Briefs, vol. 51, no. 10, pp. 505-506, 2004. 
[14] V. Milanović and M. E. Zaghloul, "Improved masking algorithm for chaotic communications systems," Electronics Letters, vol. 32, no. 1, pp. 11-12, 1996.

[15] G. Kolumban, P. M. Kennedy, and L. O. Chua, "The role of synchronization in digital communications using chaos. II. Chaotic modulation and chaotic synchronization," IEEE Transactions on Circuits \& Systems I Fundamental Theory \& Applications, vol. 45, no. 11, pp. 1129-1140, 1998.

[16] K. M. Cuomo, A. V. Oppenheim, and S. H. Strogatz, "Synchronization of Lorenz-based chaotic circuits with applications to communications," IEEE Transactions on Circuits and Systems II: Analog \& Digital Signal Processing, vol. 40, no. 10, pp. 626-633, 1993.

[17] T. Yang and L. O. Chua, "Secure communication via chaotic parameter modulation," IEEE Transactions on Circuits \& Systems I: Fundamental Theory \& Applications, vol. 43, no. 9, pp. 817-819, 1996.

[18] D. Huang, "Synchronization-based estimation of all parameters of chaotic systems from time series," Physical Review EStatistical, Nonlinear, and Soft Matter Physics, vol. 69, no. 6, Article ID 067201, 2004.

[19] F. Tang, "An adaptive synchronization strategy based on active control for demodulating message hidden in chaotic signals," Chaos, Solitons \& Fractals, vol. 37, no. 4, pp. 1090-1096, 2008.

[20] X.-J. Wu, H. Wang, and H.-T. Lu, "Hyperchaotic secure communication via generalized function projective synchronization," Nonlinear Analysis. Real World Applications, vol. 12, no. 2, pp. 1288-1299, 2011.

[21] G. Mazzini, G. Setti, and R. Rovatti, "Chaotic complex spreading sequences for asynchronous DS-CDMA. I. System modeling and results," IEEE Transactions on Circuits and Systems I: Fundamental Theory and Applications, vol. 44, no. 10, pp. 937947, 1997.

[22] T. Yang and L. O. Chua, "Chaotic digital code-division multiple access (CDMA) communication systems," International Journal of Bifurcation and Chaos in Applied Sciences and Engineering, vol. 7, no. 12, pp. 2789-2805, 1997.

[23] H.-P. Ren, C. Bai, J. Liu, M. S. Baptista, and C. Grebogi, "Experimental validation of wireless communication with chaos," Chaos: An Interdisciplinary Journal of Nonlinear Science, vol. 26, no. 8, Article ID 083117, 2016.

[24] G. M. Maggio, N. Rulkov, and L. Reggiani, "Pseudo-chaotic time hopping for UWB impulse radio," IEEE Transactions on Circuits and Systems I: Fundamental Theory \& Applications, vol. 48, no. 12, pp. 1424-1435, 2001.

[25] V. Annovazzi-Lodi and G. Aromataris, "Privacy in two-laser and three-laser chaos communications," IEEE Journal of Quantum Electronics, vol. 51, no. 7, pp. 1-5, 2015.

[26] F. Kuwashima, T. Shirao, T. Kishibata et al., "High effective generation and detection of $\mathrm{THz}$ waves using a laser chaos and a super-focusing with metal V-grooved waveguides," in Proceedings of the 40th International Conference on Infrared, Millimeter, and Terahertz Waves (IRMMW-THz '15), Hong Kong, China, August 2015.

[27] O. I. Moskalenko, A. A. Koronovskii, and A. E. Hramov, "Generalized synchronization of chaos for secure communication: remarkable stability to noise," Physics Letters, Section A: General, Atomic and Solid State Physics, vol. 374, no. 29, pp. 2925-2931, 2010.

[28] D. Ghosh, "Nonlinear-observer-based synchronization scheme for multiparameter estimation," Europhysics Letters, vol. 84, no. 4, Article ID 40012, pp. 605-609, 2008.
[29] D. Ghosh and A. Roy Chowdhury, "Lag and anticipatory synchronization based parameter estimation scheme in modulated time-delayed systems," Nonlinear Analysis: Real World Applications, vol. 11, no. 4, pp. 3059-3065, 2010.

[30] D. Huang, G. Xing, and D. W. Wheeler, "Multiparameter estimation using only a chaotic time series and its applications," Chaos. An Interdisciplinary Journal of Nonlinear Science, vol. 17, no. 2, pp. 471-516, 2007.

[31] F. Sun, L. Li, H. Peng, C. Wang, and Y. Yang, "Multiple information transmission using only one scalar chaotic time series," The European Physical Journal B, vol. 86, no. 2, article 39, 2013.

[32] C. E. Shannon and W. Weaver, The Mathematical Theory of Communication, vol. 85, no. 2, University of Illinois, Urbana University of Illinois Press, 1949.

[33] R. V. L. Hartley, Transmission of Information, The M.I.T.Pr. and John W, 1965.

[34] M. C. Mackey and L. Glass, "Oscillation and chaos in physiological control systems," Science, vol. 197, no. 4300, pp. 287-289, 1977.

[35] F. Sun, H. Peng, Q. Luo, L. Li, and Y. Yang, "Parameter identification and projective synchronization between different chaotic systems," Chaos, vol. 19, no. 2, p. 259, 2009.

[36] H. Peng, L. Li, Y. Yang, and F. Sun, "Conditions of parameter identification from time series," Physical Review E: Statistical, Nonlinear, \& Soft Matter Physics, vol. 83, no. 3, part 2, pp. 9891010, 2011.

[37] H. F. Harmuth, Transmission of Information by Orthogonal Functions, Springer, 1972.

[38] H. Yin, X. Chen, H. Yue et al., "Experimental realization of longhaul chaotic optical secure communications," in Proceedings of the 12th International Conference on Fuzzy Systems and Knowledge Discovery (FSKD '15), pp. 2112-2116, Zhangjiajie, China, August 2015. 


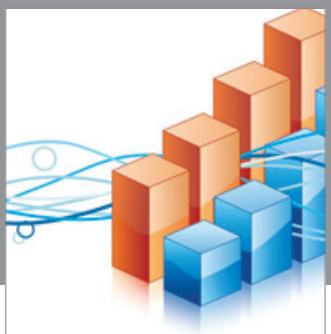

Advances in

Operations Research

vatem alat4

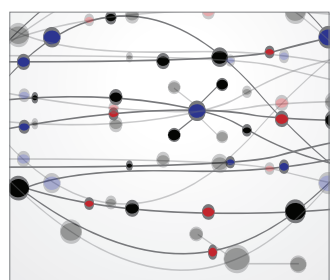

\section{The Scientific} World Journal
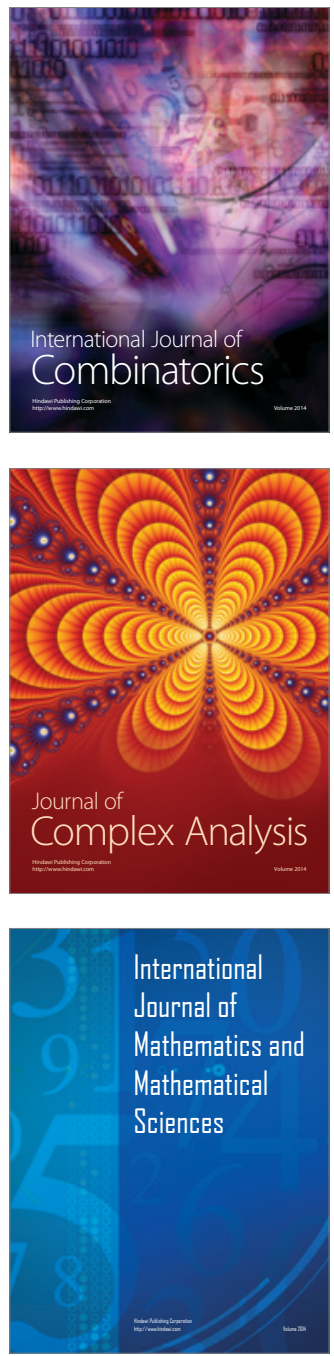
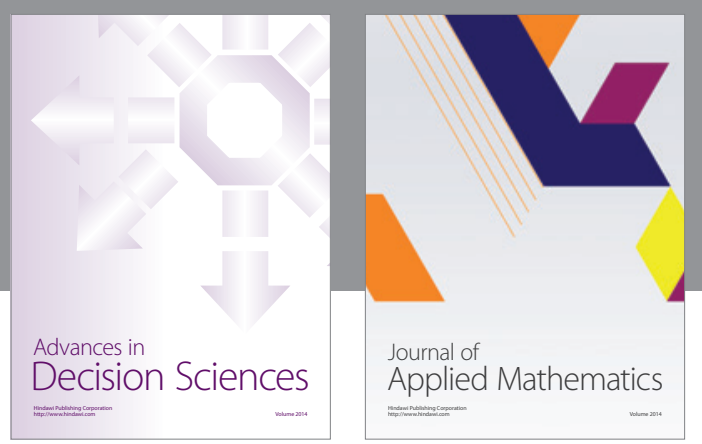

Algebra

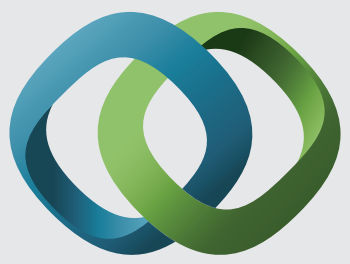

\section{Hindawi}

Submit your manuscripts at

https://www.hindawi.com
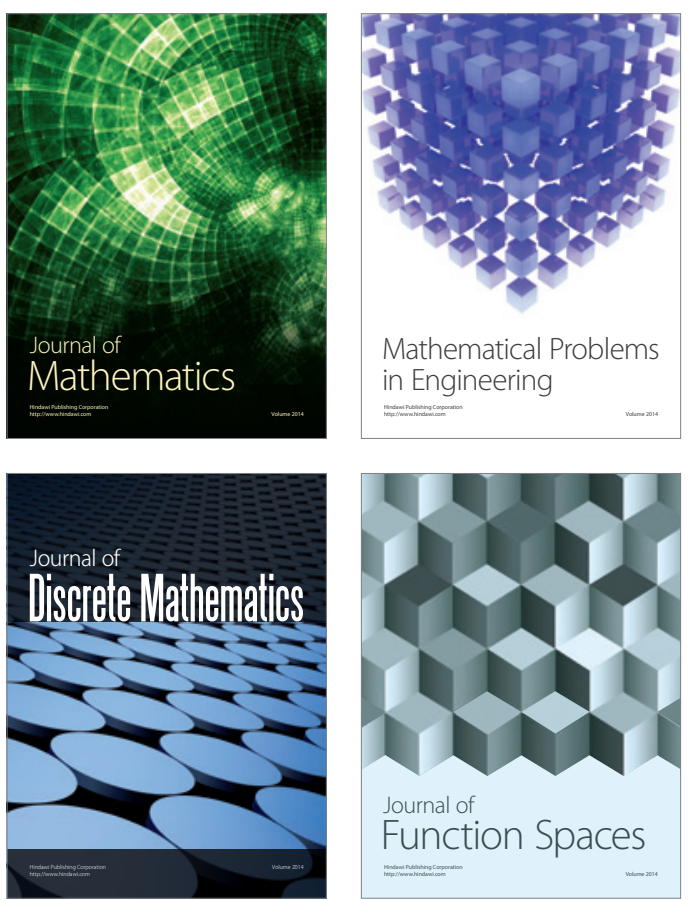

Mathematical Problems in Engineering
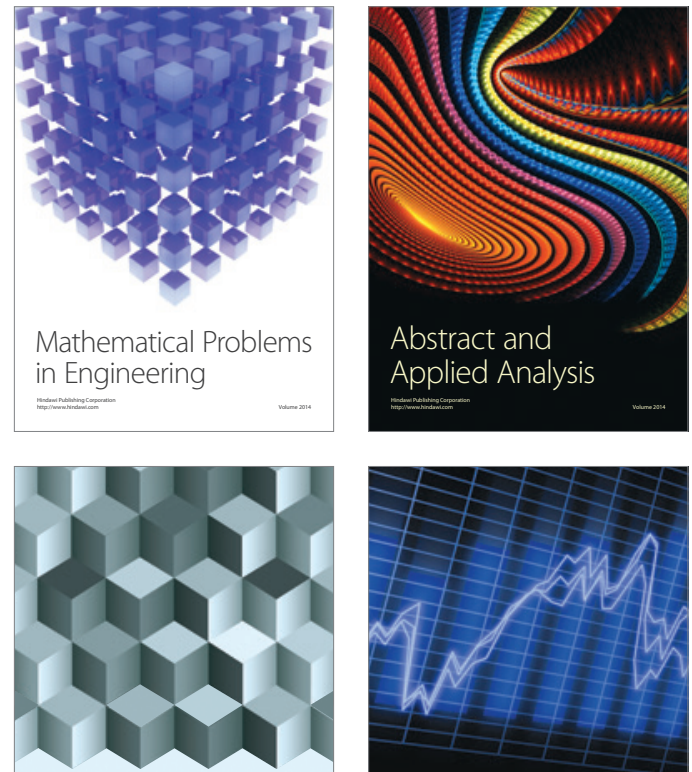

Journal of

Function Spaces

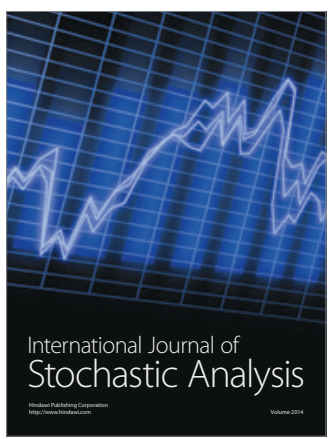

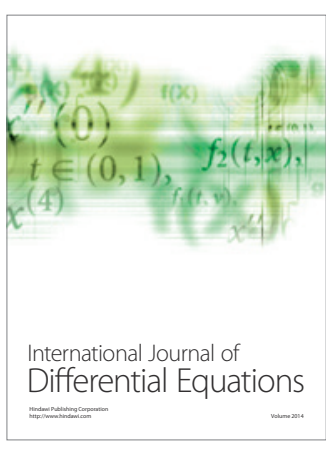
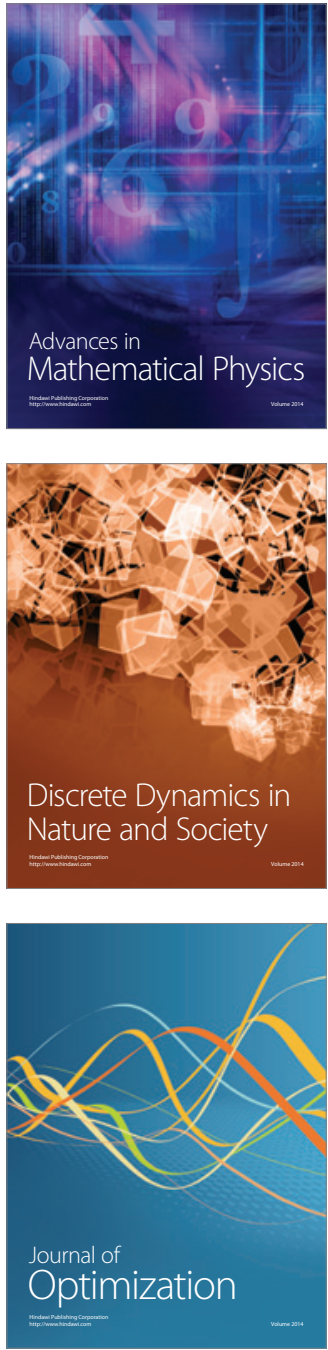